Noticias de la AEP

\title{
El lanzamiento europeo de la psicoterapia interpersonal en el X Congreso Mundial de Psiquiatría
}

\author{
J. Solé-Puig \\ Departamento de Psiquiatría, Universidad de Barcelona \\ La redefinición alemana de nuestra especialidad como psiquiatría y psicoterapia influirá profundamente en nuestra profesión en \\ toda la Unión Europea y fuera de ella. \\ C. Ballús \\ (antiguo Presidente de la Asociación de Psiquiatras Europeos)
}

Estamos completamente de acuerdo con C. Ballús. El psiquiatra del próximo siglo tendrá que ser experto en uno o más enfoques psicoterapéuticos. En el X Congreso Mundial de Psiquiatría en Madrid, en agosto de 1996, se celebró un curso y un simposio sobre Psicoterapia o Terapia Interpersonal (TIP). La TIP, creada por Gerald Klerman (1928-1992), Myrna Weissman y sus colaboradores, es una formalización hecha manual (Klerman et $a l, 1984)$ de procedimientos utilizados a menudo por psiquiatras. El Presidente de la Asociación Psiquiátrica Mundial, J. J. López-Ibor, estaba interesado en la participación de Myrna Weissman pero, por desgracia, ésta no pudo asistir a la reunión.

M. Weissman, de la Universidad de Columbia, cofundadora de la TIP, y J. C. Markowitz, de la Universidad de Cornell, continúan distinguiendo el área de Nueva York como una referencia para la TIP. J. C. Markowitz ha contribuido a unir a los profesionales europeos implicados en la TIP, lo que agradecemos.

En la sección del congreso sobre reproducción y salud mental, M. Spinelli, de la Universidad de Columbia, mostró en un ensayo clínico piloto controlado la eficacia de la TIP para mujeres deprimidas en anteparto. Esto es pertinente, pues la psico- terapia es una indicación de primera elección en las condiciones para parto.

El curso de TIP fue impartido por E. Schramm, de la Universidad de Friburgo (Alemania), que se formó en TIP con E. Frank y D. Kupfer en la Universidad de Pittsburgh, Pennsylvania, y está impulsando la investigación y difusión de la TIP de vuelta en Alemania. Se ha creado una asociación sobre TIP y se ha celebrado el primer simposio anual. En los países de habla alemana, más de 350 terapeutas han recibido formación. La autora ha publicado recientemente una amplia monografía (Schramm, 1996) sobre TIP, el primer esfuerzo europeo en el campo. Este manual de segunda generación sobre TIP incluye una minuciosa actualización de la investigación psicobiológica, la traducción de la sección de técnica operatoria en el manual de consulta (Klerman et al, 1984) y mensajes prácticos para terapeutas que aprenden TIP. Se ha planeado una traducción española del libro.

El simposio «Estado actual de la psicoterapia interpersonal en Europa» mostró que los datos de eficacia de ensayos clínicos controlados proponen a la TIP como una alternativa o complemento razonable a la medicación para pacientes con depresión mayor. También es un tratamiento prometedor para

Solé-Puig J. The European launch of interpersonal psychotherapy in the Xth World Congress of Psychiatry. Eur Psychiatry 1997; 12: 46-48. 
la bulimia nerviosa, el trastorno bipolar, la distimia, el trastorno de pánico, el trastorno límite de la personalidad, el trastorno por estrés postraumático y otras nuevas adaptaciones de la TIP en desarrollo.

El investigador en psicoterapia, C. Fairburn, de la Universidad de Oxford, puso énfasis en la bulimia nerviosa como la segunda mejor indicación de la TIP después de la depresión mayor. Su estudio anterior (Klerman y Weissman, 1993) encontró que era tan efectiva como el tratamiento dominante para esta condición (la terapia cognitivo-conductual). Se describió el ensayo multicéntrico en curso, Stanford-Oxford-Columbia, con un impresionante diseño para investigar cómo opera la TIP en la bulimia nerviosa y para establecer si diferentes tipos de pacientes responden preferencialmente a la terapia.

J. Aldenhoff y K. Müller-Popkes, ambos de la Universidad de Kiel, hicieron sus presentaciones. La primera mostraba datos empíricos de que la TIP normaliza las deficiencias de la homeostasis del calcio celular en los pacientes deprimidos, un interesante ejemplo de un efecto biológico concreto causado por psicoterapia. La segunda describía un ensayo clínico sobre insomnio primario con pacientes no medicados, en el que se probó que la TIP era más efectiva que el tratamiento de relajación progresiva, lo que apoya la idea de la TIP de combinar técnicas dirigidas al síntoma con cuestiones interpersonales que tienen que ver con el desarrollo y el mantenimiento de un trastorno específico.

M. Diéguez presentó una de las iniciativas sobre TIP que está emprendiendo el activo grupo de Madrid en torno a A. Fernández-Liria. Este último, un destacado psiquiatra español, está haciendo esfuerzos por generalizar la psicoterapia, en especial la TIP, en el sistema público de salud mental. Durante el congreso tuvo lugar la constitución de la Sociedad Española de Psicoterapia Interpersonal. Psicólogos y psiquiatras demandan mejores niveles de formación en psicoterapia, y cada vez se tiene más en cuenta la garantía de calidad en salud mental. Este es el contexto en el que se debe introducir la TIP en España (Solé-Puig, 1995) y en todo el mundo.

\section{Enseñar psiquiatría, integrar la psicoterapia}

E. Schramm y J. Solé-Puig, Presidente y Co-presidente del simposio, resumieron el estado actual de la TIP en Europa. El interés por la TIP está aumentando consistentemente entre los psiquiatras y psi- cólogos europeos. Debido a las diferencias en los sistemas de salud y el medio cultural, hay algunas desemejanzas en el uso de la TIP en comparación con los Estados Unidos. Por ejemplo, en Alemania, la TIP se aplica sobre todo como un tratamiento para pacientes internos. En España y otros países de la Unión Europea, los psiquiatras y psicólogos, abrumados de pacientes ambulatorios, tienen dificultades para proporcionar asistencia psicoterapéutica continuada. En Europa, se deben hacer esfuerzos para generalizar la práctica y la formación en psicoterapia, integrando en los currículos académicos al menos dos o tres enfoques psicoterapéuticos. Uno de ellos podría ser la TIP.

En 1992, el año de la muerte de Kerman, durante el 95 Deutsche Ärztetag, los médicos alemanes redefinieron nuestra especialidad médica como psiquiatría y psicoterapia. Es un cambio oficial en el programa de la formación psiquiátrica que hace hincapié en una integración mayor de los enfoques psicoterapéuticos en la psiquiatría clínica. En el mismo año, la Junta Europea de Psiquiatría de la Unión Europea de Médicos Especialistas apuntó armonizar la formación psiquiátrica entre los países de la unión Europea (o en Europa como una región de la Organización Mundial de la Salud). Existe una necesidad de armonizar los requisitos de formación en Europa, debido a los niveles muy diferentes de formación psiquiátrica en los respectivos países europeos. La meta es alcanzar niveles óptimos y homogéneos de formación psiquiátrica en Europa.

Los diversos países europeos muestran diferencias en la educación y la práctica psiquiátricas, en oposición a los acontecimientos en los Estados Unidos de América. Entre esas diferencias, una muy importante es el lugar que tiene la psicoterapia en la psiquiatría de cada país. Pero cada vez estamos más de acuerdo con la idea básica de que la formación en psicoterapia debería ser obligatoria en todo currículo psiquiátrico europeo.

\section{Psicoterapia: más una «herramienta» que una «escuela»}

¿Qué clases de psicoterapias deberían incluir los currículos europeos? Hasta ahora, la afirmación más repetida es que la formación en psicoterapia debería incluir al menos enfoques psicodinámicos y cognitivo-conductuales. Podemos añadir una tercera herramienta psicoterapéutica. Si el propósito principal.de 
los nuevos currículos es ofrecer un enfoque multidimensional para el diagnóstico y la terapia de los trastornos psiquiátricos sobre la base de la psiquiatría biológica, la psicoterapia y la psiquiatría social, la TIP es un enfoque que encaja bien.

El prestigio científico de la TIP en la década de 1990 es indudable. Es el enfoque psicoterapéutico más citado en las recientes publicaciones de psiquiatría biológica. De todos es conocido su éxito en el Programa de Investigación en Colaboración del Tratamiento de la Depresión del NIMH (Elkin et al, 1989) y en dos directrices prácticas importantes: una de la Asociación Psiquiátrica Americana dirigida a los psiquiatras, y el Grupo de Directrices de Depresión del Departamento de Salud y Servicios Humanos de los Estados Unidos dirigido a la asistencia primaria.

G. Klerman, M. Weissman y sus colaboradores crearon la TIP como un agente psicoterapéutico, análogo a uno psicofarmacológico, los antidepresivos. Los 4 a 6 meses de TIP para depresión son los mismos 4 a 6 meses que el tratamiento de continuación recomendado con antidepresivos. La TIP es el mejor ejemplo de una tendencia actual en el campo: estamos avanzando de las psicoterapias de escuela a psicoterapias orientadas a los trastornos. A los psicoterapeutas de hoy no les preocupan las lealtades de escuela, lo que quieren es una herramienta psicoterapéutica eficiente. La TIP podría satisfacer esa demanda.

Se suele describir la TIP como un enfoque pragmático, ateórico, no ideológico y ecléctico. Son adjetivos buenos. En él se ven las raíces psicodinámicas, aunque la posición terapéutica es la opuesta al psicoanálisis. La TIP muestra aspectos cognitivos conductuales, como las técnicas de solución de problemas y el papel activo y alentador del terapeuta. Pero los terapeutas del TIP no dan trabajo para casa a los pacientes, y no piensan en términos pavlovianos. Además, el enfoque sobre las relaciones interpersonales se solapa con la terapia familiar. Pero la TIP es fundamentalmente individual, aunque se han introducido nuevos formatos (parejas, grupos). La TIP es ecléctica no como una labor hecha de retales, sino como un modo semiestructurado de guiar al paciente durante unos meses, como una formalización de procedimientos utilizados a menudo por los psiquiatras. Es acogedora con el usuario y los psiquiatras que utilizan la TIP se sienten a gusto con ella. Por último, pero no con menos importancia, los pacientes aceptan bien la TIP. Después de la introducción de los enfoques psicodinámicos, conductual y cognitivo, la TIP es un enfoque de orientación interpersonal, de apoyo y expresivo, sin ideología, introduce la expresión de los sentimientos y las emociones en el núcleo de una psicoterapia hecha manual, y es una revolución afectiva en el campo.

La TIP, con su falta de rigidez, muestra un terreno sólido basado más en el conocimiento empírico y menos en los sistemas de creencias. La fe de escuela no debería ser un obstáculo para que la TIP mejorara con la investigación, convirtiéndose probablemente en una herramienta mejor. La TIP podría ser incluso un modelo para la investigación en el campo de la psicoterapia. Conclusión: la formación psicoterapéutica en Europa debería incluir al menos el enfoque psicodinámico, el cognitivo conductual y el interpersonal.

La reunión de la TIP durante el X Congreso Mundial de Psiquiatría acabó con un vivo debate y planes de creación de redes entre los psiquiatras y los psicólogos implicados. De debatió la creación de una futura asociación europea de TIP.

\section{BIBLIOGRAFIA}

American Psychiatric Association. Practice Guidelines for Major Depressive Disorder in Adults. Am J Psychiatry 1993; 150 (suppl 4): 1-26.

Depression Guideline Panel. Clinical Practice Guideline \#5: Depression in Primary Care, 2: Treatment of Major Depression. Rockville, MD: United States Department of Health and Human Services, Agency for Health Care Policy and Research, AHCPR publication 93-0551, 1993.

Elkin I, Shea MT, Watkins JT et al. National Institute of Mental Health Treatment of Depression Collaborative Research Program: general effectiveness of treatments. Arch Gen Psychiatry 1989; 46: 971-82.

Klerman GL, Weissman MM, Rounsaville BJ, Chevron ES. Interpersonal Psychotherapy of Depression. New York: Basic Books, 1984.

Klerman GL, Weissman MM. New Applications of Interpersonal Psychotherapy, Washington DC: American Psychiatric Press, 1993.

Schramm E. Interpersonelle Psychotherapie -zur Behandlung depressiver und anderer psychischer Störungen. Stuttgart: Schattauer, 1996.

Solé-Puig J. Psicoterapia Interpersonal (I, II). Rev Psiquiatría Fac Med Barna 1995; 22 (4): 91-9, 22 (5): $120-31$. 